\title{
Genetic Programming of Heterogeneous Ensembles for Classification
}

\author{
Hugo Jair Escalante ${ }^{1, \star}$, Niusvel Acosta-Mendoza ${ }^{1,2, \star}$, \\ Alicia Morales-Reyes ${ }^{1}$, and Andrés Gago-Alonso ${ }^{2}$ \\ 1 Instituto Nacional de Astrofísica, Óptica y Electrónica (INAOE), \\ Luis Enrique Erro No. 1, Sta. María Tonantzintla, Puebla, CP: 72840, Mexico \\ 2 Advanced Technologies Application Center (CENATAV), \\ 7a No. 21406 e/ 214 and 216, Siboney, Playa, CP: 12200, Havana, Cuba \\ hugojair@inaoep.mx, nacosta@cenatav.co.cu
}

\begin{abstract}
The ensemble classification paradigm is an effective way to improve the performance and stability of individual predictors. Many ways to build ensembles have been proposed so far, most notably bagging and boosting based techniques. Evolutionary algorithms (EAs) also have been widely used to generate ensembles. In the context of heterogeneous ensembles EAs have been successfully used to adjust weights of base classifiers or to select ensemble members. Usually, a weighted sum is used for combining classifiers outputs in both classical and evolutionary approaches. This study proposes a novel genetic program that learns a fusion function for combining heterogeneous-classifiers outputs. It evolves a population of fusion functions in order to maximize the classification accuracy. Highly non-linear functions are obtained with the proposed method, subsuming the existing weighted-sum formulations. Experimental results show the effectiveness of the proposed approach, which can be used not only with heterogeneous classifiers but also with homogeneous-classifiers and under bagging/boosting based formulations.
\end{abstract}

Keywords: Heterogeneous ensembles, Genetic programming.

\section{Introduction}

Committee-based classifiers have been studied for a considerable time within pattern recognition and machine learning [1]. The effectiveness of ensemble classifiers is widely known, in fact, the combination of experts' outputs has also been adopted in other tasks like feature selection and clustering. The underlying ensembles' principle is that by combining the outputs of (effective-enough) weak-learners that make uncorrelated mistakes, predictive models that outperform any of the individual ones can be obtained [1, 2]. Traditional models for this formulation are boosting (Adaboost [3]), and bagging (random forest [4]).

Heterogeneous ensembles are a type of committee classifiers that combine predictions of weak-learners from different nature (e.g., decision tree, neural

\footnotetext{
* Corresponding authors.
} 
networks) [5]. Intuitively, the goal is to build committee classifiers by exploiting the biases of different classifiers. Acceptable performance has been achieved by these ensembles, comparable or even better than by classical techniques [5]. The main problem of these methods is the effective selection of classification models that results in uncorrelated errors. Moreover, output normalization is a problem because different classifiers return predictions in distinct scales, e.g., having a probabilistic method and a support vector machine (SVM).

Independently of its type (homogeneous or heterogeneous), in most ensemble classification models, either a voting strategy or a linear combination of the outputs of individual classifiers is used to fuse decisions of individual classifiers [3 10]. Despite being effective, this is not necessarily the best criterion for outputs combination. For instance, a non-linear function may be preferred for complex decision surfaces. Besides, alternative fusion functions may better exploit classifiers' diversity and accuracy. Thus, regardless of individual models effectiveness/diversity, ensemble methods performance can be improved by learning the appropriate fusion function.

This paper proposes an evolutionary algorithm that aims to learn a function for combining ensemble members outputs in such a way that classification performance is maximized. A genetic program (GP) is proposed such that a population of fusion functions is evolved. Each function combines the outputs of a subset of individual classifiers. The classical linear combination approach is subsumed by this proposal. The proposed GP can automatically deal with variations in the predictions scale and can weight different classifiers outputs. Empirical results are reported using an object recognition data set. The obtained results are encouraging: the proposed method outperforms weighted and unweighted linear combination approaches. Furthermore, to the best of our knowledge, these results are the best so far obtained for the considered data set.

\subsection{Problem Statement}

Let $\mathcal{D}=\left(\mathbf{x}_{i}, y_{i}\right)_{\{1, \ldots, N\}}$ be a data set with $N$ pairs of instances $\left(\mathbf{x}_{i}\right)$ and their labels $\left(y_{i}\right)$ associated to a supervised classification problem. Without loss of generality 1 it is assumed $\mathbf{x}_{i} \in \mathbb{R}^{d}$ and $y_{i} \in\{-1,1\}$, that is a binary classification problem with numeric attributes. We denote by $g_{k}\left(\mathbf{x}_{i}\right) \in[-1,1]$ the output of classifier $g_{k}$ for instance $\mathbf{x}_{i}$, this value is associated to the confidence that $g_{k}$ has about the class of $\mathbf{x}_{i}$. Every $g_{k}$ term can be thought as a function $g_{k}: \mathbb{R}^{d} \rightarrow$ $[-1,1]$, where the predicted class for $\mathbf{x}_{i}$, denoted by $\hat{y}_{i}$, is obtained as follows: $\hat{y}_{i}=\operatorname{sign}\left(g_{k}\left(\mathbf{x}_{i}\right)\right)$.

Let $f\left(g_{1}\left(\mathbf{x}_{i}\right), \ldots, g_{L}\left(\mathbf{x}_{i}\right)\right)$ be the ensemble fusion function combining $L$ classifiers outputs $g_{\{1, \ldots, L\}}\left(\mathbf{x}_{i}\right)$ for instance $\mathbf{x}_{i}$. The most used fusion function is:

$$
f\left(g_{1}\left(\mathbf{x}_{i}\right), \ldots, g_{L}\left(\mathbf{x}_{i}\right)\right)=\frac{1}{L} \sum_{k=1}^{L} w_{k} \cdot g_{k}\left(\mathbf{x}_{i}\right)
$$

\footnotetext{
${ }^{1}$ Multiclass classification problems can be approached with multiple binary classifiers as described in Section 3 .
} 
where $w_{k}$ is the weight associated to classifier $k$. For Adaboost $w_{k}$ is iteratively obtained and it is related to the individual performance of weak learner $g_{k}$ [3]. In random forest and other ensembles $w_{k}$ is often 1 [4]. In majority vote strategies $w_{k}=1$ and $g_{k}\left(\mathbf{x}_{i}\right)=\operatorname{sign}\left(g_{k}\left(\mathbf{x}_{i}\right)\right)$. This paper tackles the problem of learning a fusion function $f^{*}\left(g_{1}(\mathbf{x}), \ldots, g_{L}(\mathbf{x})\right)$, such that its classification performance (in an unseen data set) is as highest as possible. A GP is proposed to search the functions space that is generated by using a combination of a predefined set of arithmetic operators, constants and classifiers' outputs.

\section{Related Work}

Evolutionary and bio-inspired algorithms have been widely used to support the construction of ensemble classifiers under both homogeneous and heterogeneous alternatives 6 9, 11]. The most tackled problems from the evolutionary perspective are those of $(i)$ selecting ensemble members and $(i i)$ adjusting weights in a linear combination approach. $(i)$ is a combinatoric problem, where given a set of models it is decided which one include/exclude in the ensemble [6, 7]; thus Equation (11) is reduced to find $w_{1, \ldots, L}$ with $w_{k} \in\{0,1\}$. Problem (ii) is a real-function optimization to find optimal weights for base classifiers [8, 9]; thus Equation (11) is reduced to determine $w_{1, \ldots, L}$ with $w_{k} \in \mathbb{R}$. Methods addressing (ii) subsume those approaching $(i)$. Other evolutionary methods for ensemble learning optimize base learners that compose the ensemble [12]. Although effective models are obtained through previous approaches, the fusion function is always static. The main aim in this paper is to evolve an improved fusion function.

GP has been used for ensemble learning, see [11] for a recent and comprehensive survey. Usually classifiers based on GP are used to build an ensemble [13]. Although satisfactory results have been obtained with those methods, they are limited to work with evolutionary-based classifiers, which are very often outperformed by standard classifiers from pattern recognition (e.g., SVM). A notable exception, closely related to this study proposal, is the work by Langdon et al. c.f. 10] and references therein, where authors use GP to learn ensembles (of ensembles) of binary classifiers. However, that method cannot be applied directly to multiclass problems, it uses a large number of classifiers (up to 90) and only considers two classification methods; more importantly, models structure has huge capacity; thus being highly prone to overfitting.

\section{$3 \quad$ Genetic Programming of Ensembles}

This section describes the proposed approach to learn fusion functions to combine heterogeneous classifiers outputs. First, the multiclass problem's version is formulated and second the proposed genetic program (GP) is described. 


\subsection{Learning a Fusion Function for Ensembles}

Using notation introduced in Section 1.1, a general fusion function for multiclass problems is stated as follows:

$$
f_{m}\left(h_{1}\left(\mathbf{x}_{i}\right), \ldots, h_{L}\left(\mathbf{x}_{i}\right)\right)=\frac{1}{L} \sum_{k=1}^{L} w_{k} \cdot h_{k}\left(\mathbf{x}_{i}\right)
$$

where $h_{k}\left(\mathbf{x}_{i}\right)$ is the multiclass classifier output. For a problem with $Q$-classes, $C_{1}, \ldots, C_{Q}$, each classifier returns a confidence vector per class, $h_{k}\left(\mathbf{x}_{i}\right)=$ $\left\langle h_{k}^{1}\left(\mathbf{x}_{i}\right), \ldots, h_{k}^{Q}\left(\mathbf{x}_{i}\right)\right\rangle$. Estimates $h_{k}^{j}\left(\mathbf{x}_{i}\right)$ can be obtained in different ways, e.g., the probability (resp. similarity) for the class $j$ given instance $\mathbf{x}_{i}$ according to a naïve Bayes (resp. KNN) classifier. In this work a general methodology is adopted to be used with any classifier: one-vs-rest classifiers. Thus, a binary classifier is trained per class where the $j^{t h}$ - classifier uses as positive the training examples from class $j$ and as negative the rest. In this case, $h_{k}^{j}\left(\mathbf{x}_{i}\right)$ is the confidence that the $j^{t h}$ binary classifier on that label for instance $\mathbf{x}_{i}$ is $C_{j}$.

Hence we seek for the fusion function $f_{m}^{*}$ that maximizes the classification performance on unseen data (obtained from the same distribution as the training data set). The aim of the proposed GP is to determine $f_{m}^{*}$ among the set of functions that can be built by combining the outputs of multiclass classifiers $\left(h_{1}\left(\mathbf{x}_{i}\right), \ldots, h_{L}\left(\mathbf{x}_{i}\right)\right)$.

\subsection{Genetic Programming of Fusion Functions}

GP is an evolutionary technique in which the solutions are encoded in data structures more complex than binary or real-valued vectors, as in standard genetic algorithms [14]. A population of individuals (solutions) is randomly generated, and an iterative process begins in which solutions are recombined, mutated and evaluated. Next, a subset of the best solutions are kept for the next generation. The best solution found through the iterative process is returned.

Obtaining a fusion function for heterogeneous ensemble learning via GP requires the codification of a combination function (a solution) as a tree where leaf nodes correspond to classification models outputs augmented with constants (to incorporate a weighting factor). Non-leaf nodes are taken from the following operators: $\left\{+,-, \times, \div{ }^{2}, \sqrt{,} \log _{10}\right\}$, these are the ones typically used in GP. An individual is shown in Figure 1.

The GP input is the predictions set obtained by the considered classifiers $\left(h_{1}\left(\mathbf{x}_{i}\right), \ldots, h_{L}\left(\mathbf{x}_{i}\right)\right)$ in a training data set $\mathcal{D}$. Specifically, every instance is classified in $\mathcal{D}$ via 10 -fold cross-validation with each classifier, and these are the GP inputs; that is, we have a single value for each instance and classifier obtained when the instance belongs to the test partition in 10-fold cross-validation. We proceed in this way because we wanted to avoid overfitting as much as possible. The following classifiers are taken from the CLOP toolbox: random forest, SVM, klogistic, linear-kridge, non-linear kridge, $1 N N$, 3NN, naïve Bayes, gkridge, and 

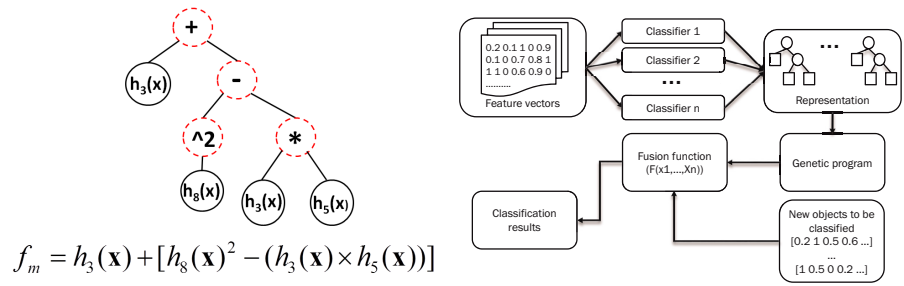

Fig. 1. GP ensemble: Individual sample (left), general scheme (right)

neural network. The GP evolves and returns a fusion function $\left(f_{m}^{*}\right)$ that is evaluated on (unseen) test data. The general diagram of the proposed approach is shown at the right in Figure 1, During the search process, the fitness value of every function $f_{m}$ is calculated by evaluating the performance of the tree's induced function: first, the predicted class per instance $\mathbf{x}_{i}$ is calculated as follows: $\hat{y}_{i}=\arg \max _{Q} f_{m}\left(h_{1}\left(\mathbf{x}_{i}\right), \ldots, h_{L}\left(\mathbf{x}_{i}\right)\right)$, which is simply the index of the class with the maximum confidence; next, $f_{m}$ predictive performance is assessed with standard measures to determine its fitness.

Two fitness functions are used: (1) accuracy, and (2) $f_{1}$-measure. The latter computes the balance between precision and recall per class; $f_{1}$-measure's average among classes is herein used. $f_{1}$ is more informative when data sets are imbalanced. The GP is implemented on the GPLAP2 framework. Standard crossover and mutation operators are adopted. The population is initialized with the ramped-half-and-half formulation.

\section{Experiments and Results}

For experimentation the SCEF data set is used, it is associated to an object recognition problem. This data set is challenging and has been previously used for evaluating heterogeneous ensemble selection methods [12]. The data set comprises 6244 image-regions represented by 737 attributes each (MPEG7/wavelet descriptors) and 10 classes. The data set is divided in two subsets: 3615 images for testing, and 2629 for training. Table1 (left) shows the distribution of training and testing examples per class.

Empirical results of two GP settings, called EGSP and EGG, are reported. In EGSP only the sum operator is used, whereas in EGG all previously described operators are used. Thus, EGSP resembles the standard approach to learn weights and select ensemble members [7 9]. Results obtained by a standard ensemble (EVP) are also presented, i.e., the fusion function from Equation (2) with $w_{k}=1$. For the three ensembles, results obtained by the GP are also reported when using only the top 5 classifiers with better performance in the training data; in order to determine how the accuracy of individual members affects GP-based ensembles performance.

\footnotetext{
2 http://gplab.sourceforge.net/
} 
Table 1. Left: Description of the data set used for experimentation. Right: Results obtained by individual classifiers in terms of accuracy/ $f-1$ measure.

\begin{tabular}{|c|c|c|c|c|c|c|c|c|c|c|c|}
\hline \multicolumn{6}{|c|}{ Data set description } & \multicolumn{6}{|c|}{ Perf. individual classifiers. } \\
\hline Class & Train & $\overline{\text { Test }}$ & Class & Train & Test & Model & Acc. & $f_{1}$ & Model & Acc. & $f_{1}$ \\
\hline Building & 280 & 450 & Foliage & 506 & 581 & $\mathrm{RF}$ & 90.7\% & $\overline{\mathbf{7 9 . 3} \%}$ & SVM & $55.1 \%$ & $49.9 \%$ \\
\hline Mountain & 203 & 349 & & 43 & 129 & logistic & $70.6 \%$ & $62.8 \%$ & Kridge-l & $13.64 \%$ & $2.4 \%$ \\
\hline Road & 89 & 127 & Sand & 208 & 273 & Kridge-n & $74.7 \%$ & $63.1 \%$ & $1 \mathrm{NN}$ & $3 \%$ & $60.1 \%$ \\
\hline Sea & 325 & 338 & Sky & 461 & 664 & $3 N N$ & $69.1 \%$ & $57.4 \%$ & N.Bayes & $26.5 \%$ & $21.6 \%$ \\
\hline Snow & 43 & 129 & SailingBoat & 39 & 70 & Gkridge & $20.6 \%$ & $3.421 \%$ & Neural N. & $55.8 \%$ & $37.7 \%$ \\
\hline
\end{tabular}

Table 1 (right) shows the performance obtained by individual classifiers in terms of accuracy and $f_{1}$-measure. Random forest significantly outperforms other classifiers. Thus, it is expected that the GP selects the best fusion function from individual classifiers. Table 2 shows the average and standard deviation after 10 runs obtained by the three GP ensemble variants, using 50 individuals and 100 generations per execution. The proposed ensemble variants outperform significantly the raw-fusion function (EVP) in terms of both measures with differences between $40-50 \%$. GP-ensembles even outperformed EVP when using the top- 5 models. This shows the limitations of the raw fusion function for heterogeneous ensembles.

All GP ensembles outperform the best individual classifier. The improvement for both performance metrics is small for all methods but for EGG. Improvements of more than $1.5 \%$ and $6 \%$ are obtained by EGG with respect to the best individual classifier, in terms of accuracy and $f_{1}$ measure, respectively. EGG is able to find very effective fusion functions for heterogeneous classifiers, even when most models performance is low. Moreover, a $6 \%$ improvement in $f_{1}$-measure is significant when persists across classes, because it focuses on the average performance over classes.

The best results are obtained by the EGG ensemble, i.e., using all operators and classifiers. Using more operators in the GP might allow to obtain better fusion functions. Moreover, the GP has more selection options because it uses all classifiers, which explains the improvement over EGG-Top 5.

The best result in Table 2 improves by more than $10 \%$ previously reported accuracy for the same data set $(81.49 \%)$ [12]. In [12], authors did not optimize the decision threshold thus the ROC curve area (AUC) is also reported. Comparing the best individual AUC (98.44) with the best result reported in [12] (94.05), an improvement of more than $4 \%$ is still achieved. These results, to the best of our knowledge, are the best ones so far reported for the SCEF data set.

The EGG performance is evaluated using different population sizes and number of generations. Figure 3 reports the average and standard deviation after 10 runs for different population sizes and 100 generations (left) and different number of generations and 50 individuals (right). Every measure's performance is higher when optimizes itself (e.g., accuracy when optimizing accuracy). Our proposal is somewhat robust to parameters variations, performance differences by distinct settings are very closed to each other. The number of generations seems to have a slightly higher impact in EGG performance than the population 
Table 2. Different strategies performance when optimizing accuracy (top) and $f_{1}$ (bottom). EVP: raw fusion; EGSP: GP using only sums; EGG: proposed GP.

\begin{tabular}{|r|c|c|c|c|c|c|}
\hline \multicolumn{7}{|c|}{ Results obtained when optimizing accuracy. } \\
\hline & EVP & EVP-Top5 & EGSP & EGSP-Top5 & EGG & EGG-Top5 \\
\hline \hline Acc. & $31.5 \%$ & $81.4 \%$ & $90.8 \%(0.001)$ & $91.1 \%(0.002)$ & $\mathbf{9 2 . 3} \%(\mathbf{0 . 0 0 2})$ & $91.2 \%(0.001)$ \\
\hline$f_{1} \cdot$ & $27.2 \%$ & $71.9 \%$ & $80.3 \%(0.007)$ & $80.4 \%(0.006)$ & $\mathbf{8 5 . 2} \%(\mathbf{0 . 0 0 4})$ & $80.7 \%(0.001)$ \\
\hline
\end{tabular}

\begin{tabular}{|c|c|c|c|c|c|c|}
\hline \multicolumn{7}{|c|}{ Results obtained when optimizing $f_{1}$ (macro-average). } \\
\hline & EVP & EVP-Top5 & EGSP & EGSP-Top5 & EGG & EGG-Top5 \\
\hline & & & & & & $\overline{91.2}$ \\
\hline & $27.2 \%$ & $71.9 \%$ & $80.4 \%(0.001)$ & $80.4 \%(0.001)$ & $85.3 \%(0.003)$ & $80.545 \%(0.003)$ \\
\hline
\end{tabular}
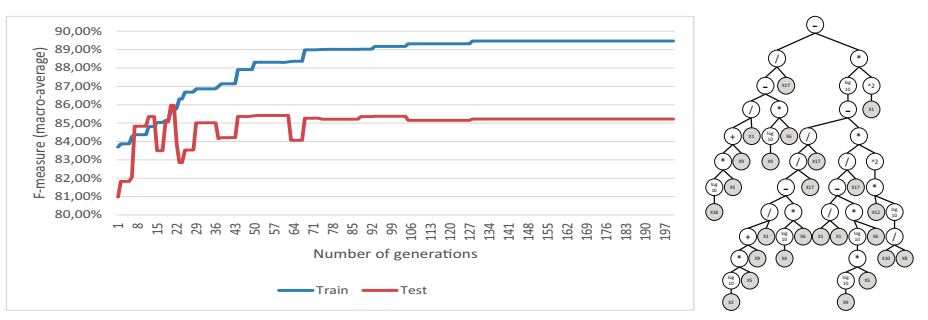

Fig. 2. EGG's best $f_{1}$ training and test values per generation (left). Evolved fusion function, shaded nodes represent classifiers' outputs (right).
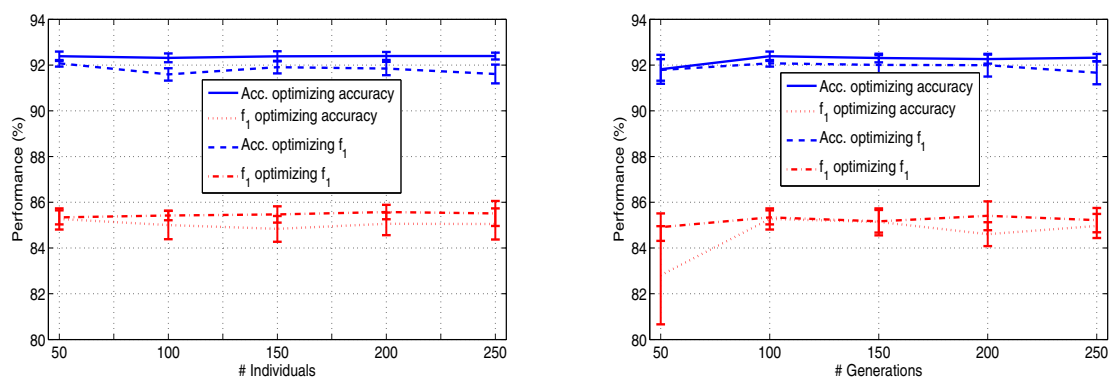

Fig. 3. Parameter selection results varying: the number of individuals (left), the number of generations (right)

size. The best configuration in terms of both performance metrics is 200 individuals and 100 generations (92.385\% and $85.57 \%$ ). A small number of iterations is preferred because a large number would overfit solutions. For example, Figure 2 shows the training and test performance of EGG for the best solution every generation for 200 individuals during 200 generations. After $\approx 30$ iterations the GP starts overfitting, and although the fitness performance keeps improving $\left(f_{1}\right.$ in this case), test set performance no longer improves and even degrades. The resultant individual after 200 generations is fairly complex (Figure 2, right). Therefore, special attention must be paid to avoid EGG overfitting. 


\section{Conclusions}

A novel GP approach to learn fusion functions for heterogeneous ensembles was proposed. Its main objective is to search the fusion-functions space generated through an arithmetic operators set. Empirical results on a challenging data set were presented and significant performance improvement over previous work $(10 \%)$ was achieved. The proposed GP outperformed the best individual model, a raw-ensemble and other variants which optimize models selection and weights. Several research directions were identified: a full experimental study on benchmark data considering parameter selection; adapting the proposed GP to homogeneous ensembles; analytical comparison to other GP-based ensembles.

\section{References}

1. Dietterich, T.: Ensemble methods in machine learning. In: Kittler, J., Roli, F. (eds.) MCS 2000. LNCS, vol. 1857, pp. 1-15. Springer, Heidelberg (2000)

2. Kuncheva, L., Whitaker, C.: Measures of diversity in classifier ensembles and their relationship with the ensemble accuracy. Mach. Learn. 51(2), 181-207 (2003)

3. Freund, Y., Schapire, R.E.: A decision-theoretic generalization of on-line learning and an application to boosting. J. Comput. Syst. Sci. 55, 119-139 (1997)

4. Breiman, L.: Random forest. Mach. Learn. 24(2), 123-140 (2001)

5. Bian, S., Wang, W.: On diversity and accuracy of homogeneous and heterogeneous ensembles. International Journal of Hybrid Intelligent Systems 4, 103-128 (2007)

6. de Oliveira, D., Canuto, A., De Souto, M.C.P.: Use of multi-objective genetic algorithms to investigate the diversity/accuracy dilemma in heterogeneous ensembles. In: Proc. of IJCNN, pp. 2339-2346 (2010)

7. Park, C., Cho, S.: Evolutionary computation for optimal ensemble classifier in lymphoma cancer classification. In: Zhong, N., Raś, Z.W., Tsumoto, S., Suzuki, E. (eds.) ISMIS 2003. LNCS (LNAI), vol. 2871, pp. 521-530. Springer, Heidelberg (2003)

8. Macaš, M., Gabrys, B., Ruta, D., Lhotská, L.: Particle swarm optimisation of multiple classifier systems. In: Sandoval, F., Prieto, A.G., Cabestany, J., Graña, M. (eds.) IWANN 2007. LNCS, vol. 4507, pp. 333-340. Springer, Heidelberg (2007)

9. Yang, L., Qin, Z.: Combining classifiers with particle swarms. In: Wang, L., Chen, K., S. Ong, Y. (eds.) ICNC 2005. LNCS, vol. 3611, pp. 756-763. Springer, Heidelberg (2005)

10. Langdon, W.B., Barrett, S.J., Buxton, B.F.: Combining decision trees and neural networks for drug discovery. In: Foster, J.A., Lutton, E., Miller, J., Ryan, C., Tettamanzi, A.G.B. (eds.) EuroGP 2002. LNCS, vol. 2278, pp. 60-70. Springer, Heidelberg (2002)

11. Espejo, P., Ventura, S., Herrera, F.: A survey on the application of genetic programming to classification. IEEE T. Syst. Man. Cyb. C 40(2), 121-144 (2010)

12. Escalante, H.J., Montes, M., Sucar, L.E.: Ensemble particle swarm model selection. In: Proc. of IJCNN, pp. 1-10 (2010)

13. Bhowan, U., Johnston, M., Zhang, M., Yao, X.: Evolving diverse ensembles using genetic programming for classification with unbalanced data. IEEE Transactions on Evolutionary Computation 17(3), 368-386 (2013)

14. Langdon, W.B., Poli, R.: Foundations of Genetic Programming. Springer (2001) 